\title{
ROUGHNESS DISCRIMINATION IN CATS WITH DORSAL COLUMN LESIONS
}

\author{
P. J. K. DOBRY AND KENNETH L. CASEY
}

Department of Physiology, University of Michigan Medical School, Ann Arbor, Mich. 48104 (U.S.A.)

(Accepted March 29th, 1972)

\section{INTRODUCTION}

The anatomical and functional integrity of the dorsal columns (DC) of the spinal cord has generally been considered essential for the preservation of refined somesthetic spatial discriminative capacity ${ }^{17}$. Recent experimental studies ${ }^{6,11,12,18}$ and clinical observations $\mathrm{s}^{2,3}$ have prompted challenges ${ }^{24}$ to this traditional view.

In dogs, a conditioned reflex to light touch (air puff) of the hindlimb remained after a thoracic DC lesion ${ }^{18}$. Cats were still able to make crude localizations (forearm vs. hindleg) of cutaneous stimuli following bilateral ablation of the somatosensory cortex and the dorsal columns ${ }^{6}$. Kitai and Weinberg ${ }^{11}$ were able to train cats with cervical DC lesions to discriminate roughness, although these animals were slower to learn than normal cats. In humans ${ }^{2,3,19}$ and monkeys ${ }^{12} \mathrm{DC}$ lesions caused only transient disturbances in touch and two-point discrimination, and, in humans, graphesthesia.

Similar observations also lead to questioning the view that the dorsal columns are essential for position sense and fine, coordinated movement ${ }^{17}$. After DC lesions, monkeys showed no loss in limb position sense ${ }^{22}$ or ability to detect movement of their digits ${ }^{21}$, and only a temporary loss of ability to discriminate weights ${ }^{4,5}$. In one study, unilateral DC section in humans caused only transient disturbances in stereognosis and appreciation of passive movement ${ }^{3}$. Melzack and Bridges ${ }^{15}$ have observed that near-total, bilateral DC lesions significantly impair the natural, coordinated motor behavior of cats and Gilman and Denny-Brown ${ }^{9}$ have described dramatic effects of DC lesions on muscular coordination, locomotion, and general behavior in the monkey. Mettler and Liss ${ }^{16}$, however, observed no consistent deficits after total bilateral DC lesions.

Behavioral studies of the effect of lesions are necessarily limited by the range of lesion sizes produced and the variety of behavioral observations which can be made. In this report, we present evidence that only lesions destroying over $90 \%$ of the total cervical DC cross-sectional area result in impairment of motor and sensory 

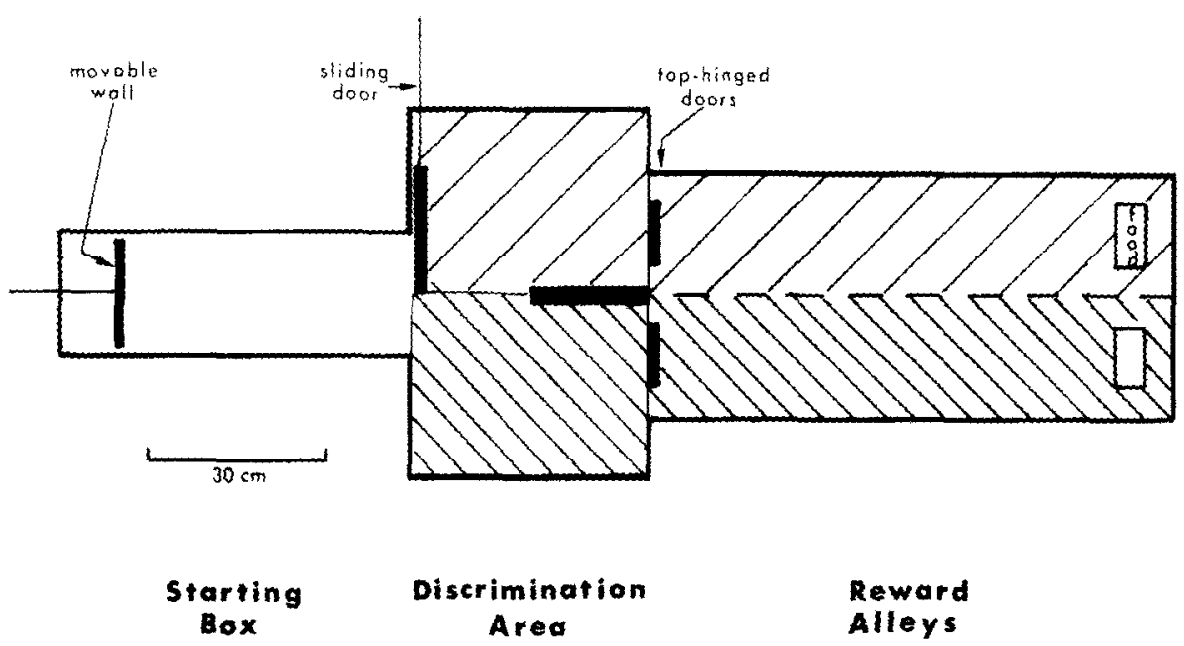

Fig. 1. Roughness-discrimination training box.

function as determined by neurological testing and the learning and retention of a roughness-discrimination task.

\section{METHODS}

Surgical procedures. The dorsal columns of 15 cats, 2 months to several years in age, were sectioned at the caudal end of the first cervical segment of the spinal cord. A laminectomy of the first vertebra was performed aseptically during sodium pentobarbital anesthesia and the dorsal columns sectioned bilaterally with a fine scalpel. The dura was repositioned and covered with a plastic film before approximation of the muscles and skin. The wound was cleansed with alcohol and covered with nitrofurazone ointment; the cats received routine injections of antibiotics (oxytetracycline hydrochloride and sulfadimethoxine) for several days. Postoperative recovery periods of 2 weeks to 2 years were allowed before the cats were used in the roughnessdiscrimination training, after which the lesions were examined histologically.

Behavioral training. Fourteen blindfolded adult cats ( 4 normal and 10 with dorsal column lesions) were trained in a modified Grice box (Fig. 1). Blindfolds were constructed according to a design of Mr. Stephen Fish, Research Assistant at Sonoma State Hospital, Eldridge, Calif. A central wall partially divided the discrimination area into halves with sandpaper floors of different roughness, extending into the corresponding reward alleys. The sandpaper discriminanda (flint paper by $3 \mathrm{M}$ or Carborundum) had the following unit numbers: extra coarse 36 ; medium 80 ; fine 150; extra fine 220. 'Smooth' paper was the reverse side of extra coarse paper. Each cat was allowed several days to become accustomed to the mask, to learn to wait in the starting box 15 $45 \mathrm{sec}$ until the sliding door opened, and to open the top-hinged doors by pressing against them with the head. In the training period, the extra coarse sandpaper and food were placed in only one reward alley and one-half of the discrim- 
ination area; the corresponding areas were covered with smooth paper and contained no food. The reward was a mixture of cat food and evaporated milk. The cats were deprived of food overnight before each session. Each cat received an average of 6 days of training per week at 20 trials per day.

Gellermann schedules $^{8}$ were used to select the sequence for the right-left position of the positive discriminandum. Occasionally, a preference for the right or left side would develop. This was corrected within 5-100 trials by placing the extra coarse sandpaper and food on the non-preferred side and sometimes locking the preferred door. After the cat had learned the discrimination between the extra coarse sandpaper and the smooth surface (criterion: at least $85 \%$ correct on 4 of 5 consecutive days), the task was changed to the next most difficult level (extra coarse/ smooth, extra coarse/extra fine, extra coarse/fine, and extra coarse/medium).

Control for nonsomatic inputs. To show that the task was performed with somatosensory cues, 20 control trials were given the day after passing each criterion. Still blindfolded, the cat wore rubber or knitted boots on all 4 feet, invariably resulting in decreased performance. An accumulated record of 1140 trials shows $50.3 \%$ correct. The cats were under observation continually. They did not touch their noses or whiskers to the sandpaper and their performance level did not vary according to whether food was placed in the reward alley before or after each choice was made.

Histology. Alternate sections of the spinal cord and brain stem of all cats were examined histologically after Nissl cresyl violet or Weil staining. The area of fiber degeneration was identified by: (1) the lack of normal staining reaction (blue-black) for myelin, and $(2)$ microscopic examination (magnification $\times 430$ ) for myelin

\section{TABLE I}

BILATERAL DORSAL COLUMN LESION SIZES, EXPRESSED AS A PERCENTAGE OF THE TOTAL DC CROSS-SECTIONAL AREA AT THE FIRST CERVICAL SEGMENT, AND OF THE MEDIAL 65\% OF THE DC AT THE SAME LEVEL

\begin{tabular}{lcc}
\hline Cat & \% of total DC & \% of medial $65 \%$ of $D C$ \\
\hline L2 & 2 & 3 \\
L11 & 11 & 17 \\
L38 & 38 & 51 \\
L57 & 57 & 71 \\
R69 & 69 & 87 \\
L71 & 71 & 77 \\
L76 & 76 & 75 \\
L82 & 82 & 89 \\
R82 & 82 & 92 \\
L86 & 86 & 89 \\
R86 & 86 & 92 \\
L90 & 90 & 93 \\
L97 & 97 & 97 \\
L100 & 100 & 100 \\
R100 & 100 & 100 \\
Lz100 & 100 & 100 \\
DL & 3 & 0 \\
- & & \\
\hline
\end{tabular}




\section{8

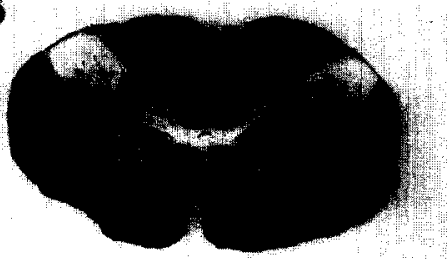

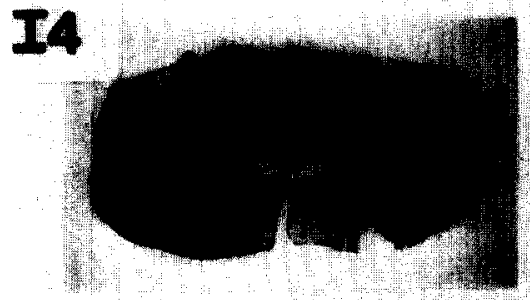
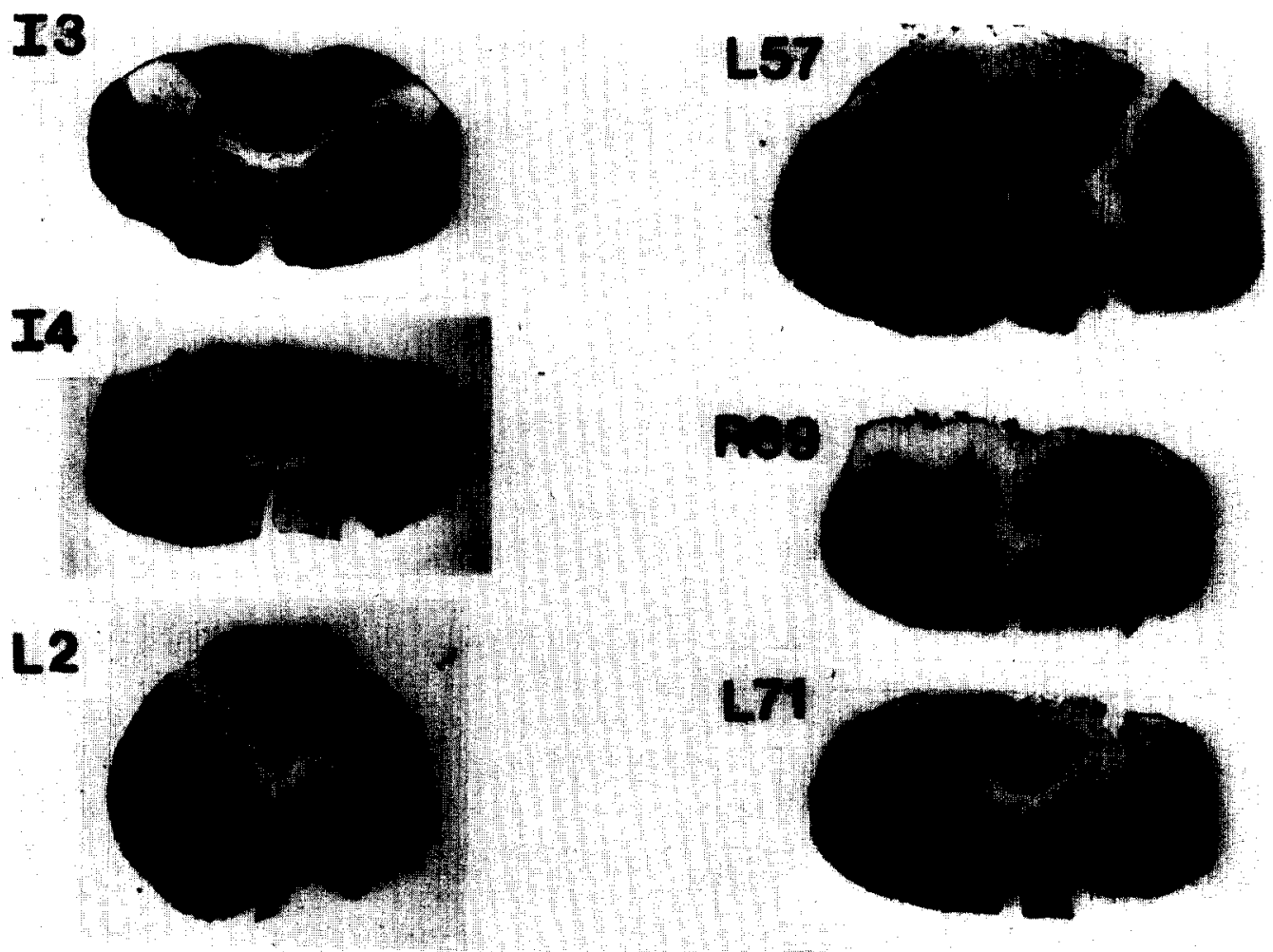

\section{L11}
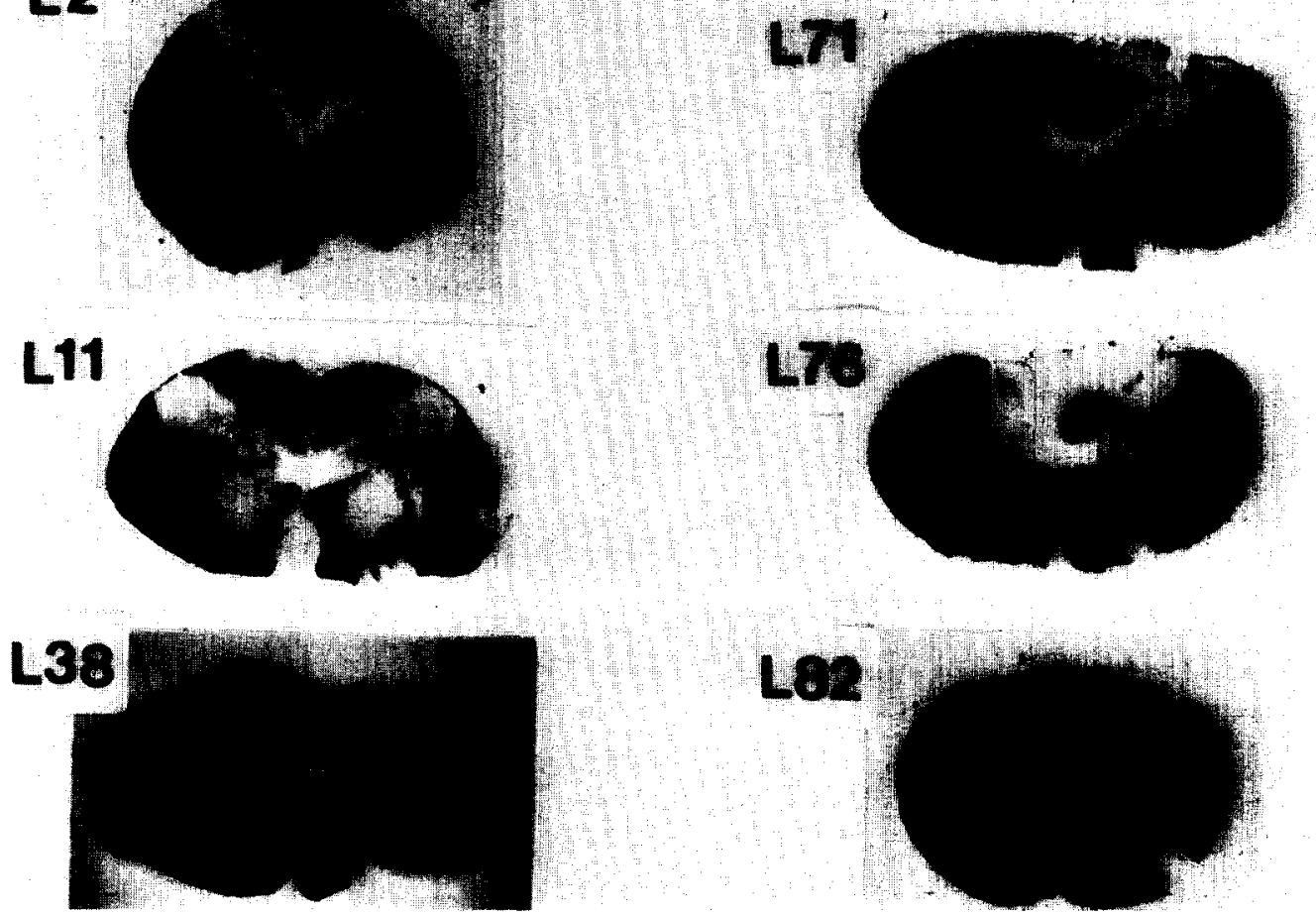

Fig. 2. Weit-stained cervical sections from all cats having surgery, whether chronic DC lesion, dorsolateral lesion, or sham operation. India ink applied to the dorsal surface of the spinal cord before sectioning appears as a black outline on the dorsal columns. For each cat the number of days between the most recent surgery and the date of death is as follows: cat 13,85 days; $\mathrm{I} 4,83$; L2, 53; L11, 784; $\mathrm{L} 38,42$ (this is the date for a sham operation; the chronic DC lesion was made 699 days before death); L57, 535; R69, 78; L71, 498; L76, 644; L82, 30; R82, 46; L86, 566; R86, 97; L90, 709; L97, 723; L100, 613; R100, 119; Lz100, 51; DL, 566. 


\section{R82}
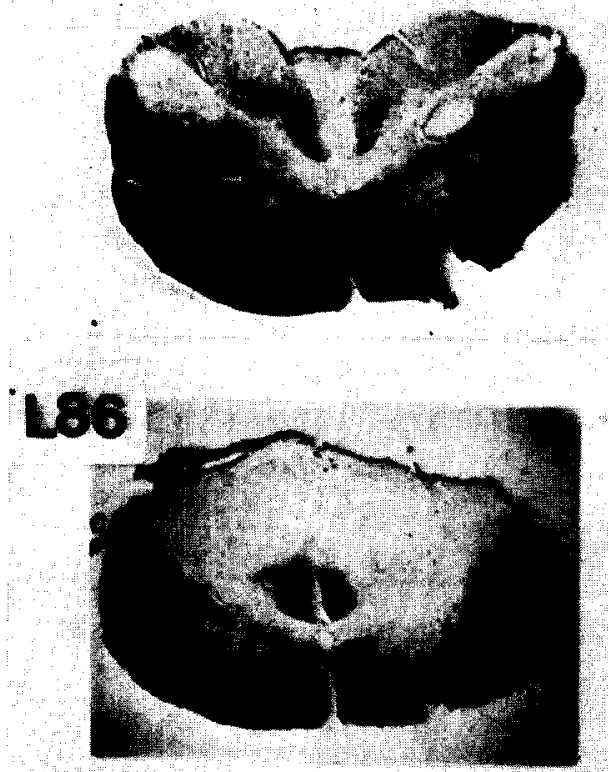

\section{Ros}
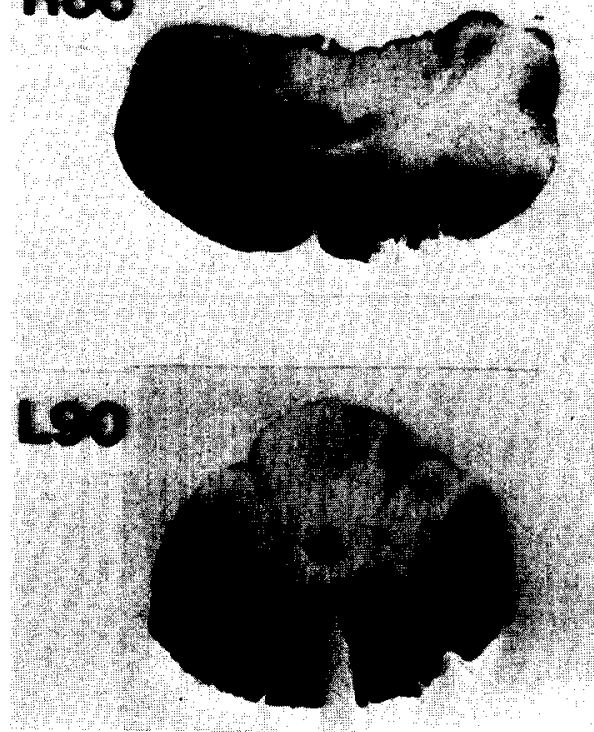
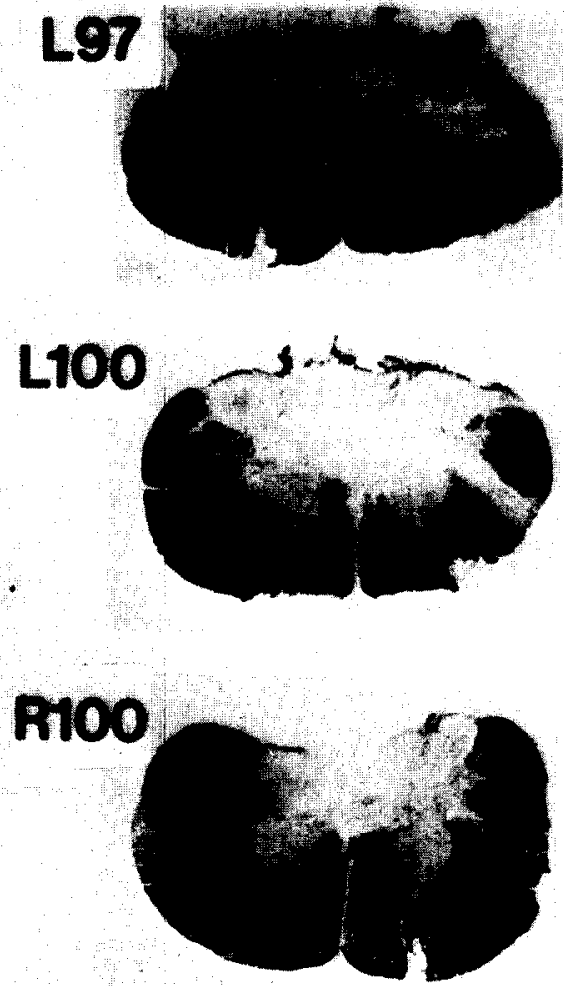

4200

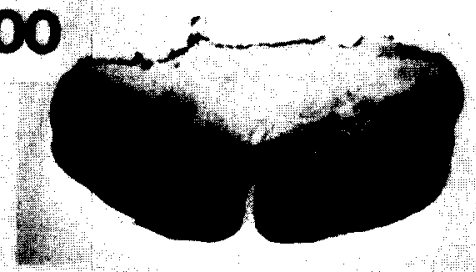

DL

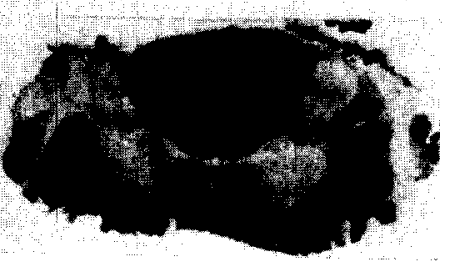


remnants in the place of circlets of normal myelin in cross-section, This was measured with a planimeter from a tracing of Weil-stained cross-sections (magnified $\times 20.8$ ) immediately rostral to the original cut.

In the text, lesion size is expressed as the percentage of the total cross-sectional DC area which had degenerated at the $\mathrm{C}_{1}$ level. However, sensory fibers entering the cord rostral to the sixth cervical segment are not involved in the discrimination task because they innervate the head, neck, pectoral girdle, and upper $\operatorname{arm}^{10}$. At the level of the first cervical segment, DC fibers from the first 5 cervical segments occupy the lateral $35 \%$ of the DC as estimated from planimetric measurements of the data obtained by $\mathrm{Liu}^{13}$ in cats and by Walker and Weaver ${ }^{23}$ in monkeys. The estimated effective lesion size is therefore presented in Table I as a percentage of a wedgeshaped area forming the medial $65 \%$ of the DC.

\section{RESULTS}

Each cat is identified by a letter and a number. Cats without lesions are designated by an I and cats with dorsal column lesions by $\mathrm{L}$ or $\mathrm{R}$, the number referring to the percentage of DC cross-sectional area damaged. DL is the cat with only a dorsolateral lesion. Fig. 2 shows the relevant histology.

\section{Neurological examinations}

Seven normal and 17 experimental cats were examined for response to pinching, righting reaction, visual and tactile placing, landing when dropped 2 feet, walking a 1-in. edge, and open field behavior with and without blindfold. Only the cats with DC lesions of at least $90 \%$ continued to show unequivocal neurological deficits when tested one month or more postoperatively and these were limited to tactile placing, edgewalking, landing, and open field behavior without blindfold. In the open field, the deficit ranged from a relatively subtle loss of smooth, coordinated walking or running to a wide-based gait with a characteristic tendency to stand or even walk on the dorsum of a paw, often seemingly unaware of this unusual posture. These animals also stumbled upon landing, hitting the nose, chin, or abdomen on the floor. Their feet frequently slipped off a 1 -in. edge and they responded erratically in tactile placing, only in the hindlimbs. In all other tests, they could not be distinguished from intact cats or those with less extensive DC lesions. All other cats, including two with $86 \%$ lesions, appeared normal in neurological examination.

\section{Roughness discrimination: animals trained postoperatively}

Seven cats ( 2 intact and 5 with 11-100\% of dorsal columns damaged) were trained under identical circumstances until they learned all 4 of the roughness discriminations or failed to meet criterion on one of the tests. After the first task, for which they were allowed as many trials as needed to pass the criterion (at least $85 \%$ correct for at least 4 of 5 consecutive days), they were allowed up to 600 trials to pass criteria on each of the next 3 tasks. 
TABLE II

THE EFFECTS OF DC LESIONS ON THE ACQUISITION OF ROUGHNESS-DISCRIMINATION SKILL

The number of trials required for each cat to perform at criterion level (at least $85 \%$ correct for 4 of 5 consecutive days) for each discrimination (extra coarse sandpaper $v s$. the grade of sandpaper listed).

\begin{tabular}{|c|c|c|c|c|}
\hline Cat & Smooth & Extra fine & Fine & Medium \\
\hline \multicolumn{5}{|l|}{ Intact } \\
\hline I1 & 220 & 100 & 100 & 100 \\
\hline 12 & 599 & 80 & 100 & 100 \\
\hline I3 & 600 & 160 & $*$ & $*$ \\
\hline I4 & 1022 & 140 & $*$ & $*$ \\
\hline \multicolumn{5}{|c|}{$\begin{array}{l}\text { Cats with chronic dorsal } \\
\text { column lesions }\end{array}$} \\
\hline L11 & 940 & 140 & 120 & - \\
\hline L38 & 350 & 100 & $*$ & $*$ \\
\hline L71 & 509 & 220 & 80 & 80 \\
\hline L86 & 560 & 80 & 100 & 340 \\
\hline L97 & 732 & - & & \\
\hline L100 & 1592 & - & & \\
\hline
\end{tabular}

- The cat was unable to perform at criterion within the 600 trials allowed.

* I3, I4, and L38 were not tested, instead, they were used in another experiment discussed in the text.

All 3 of the cats which failed discrimination tasks had DC lesions (Table II). Among these were the two with the largest DC lesions (97\% and 100\%). L97 and L100 were slow to improve in accuracy (Fig. 3) and never passed the second test. Another DC cat with a lesion of only $11 \%$ (L11) began almost as poorly, and passed 3 tests but not the fourth. The variability among DC cats may reflect some of the variability found in the learning rates of normal animals. Indeed, during the first two tasks, half of the intact cats required more trials to pass the criteria than half of the DC cats (Table II). However, the only cat which required more trials on the first task than the slowest intact cat (I4) was the cat with a total DC lesion; he required $60 \%$ more trials than I4.

For the DC cats which did not fail any tasks, the number of trials required to pass the second, third, and fourth tests was comparable to the number required by the intact cats, excepting L86 at the most difficult task. The performance of the group of DC cats after 600,1200 , and 1800 trials is not significantly different ( $t$-test) from the performance of the group of intact cats (Fig. 3). The striking difference between intact and DC cats is the failure of 3 of the DC cats to pass all of the tests.

When intact and DC cats are compared, there is no significant difference in the cumulative number of trials to criterion in any of the 4 tasks. However, the cats with the largest lesions (L97 and L100) required more trials than the combined group of intact and other DC cats to reach criterion on the second ( $t=5.4$, one-tailed test, $P<0.01)$, third $(t=7.4, P<0.001)$, and fourth $(t=3.5, P<0.01)$ tasks. These statistical comparisons include: (1) the additional trials (not shown in Table II) re- 


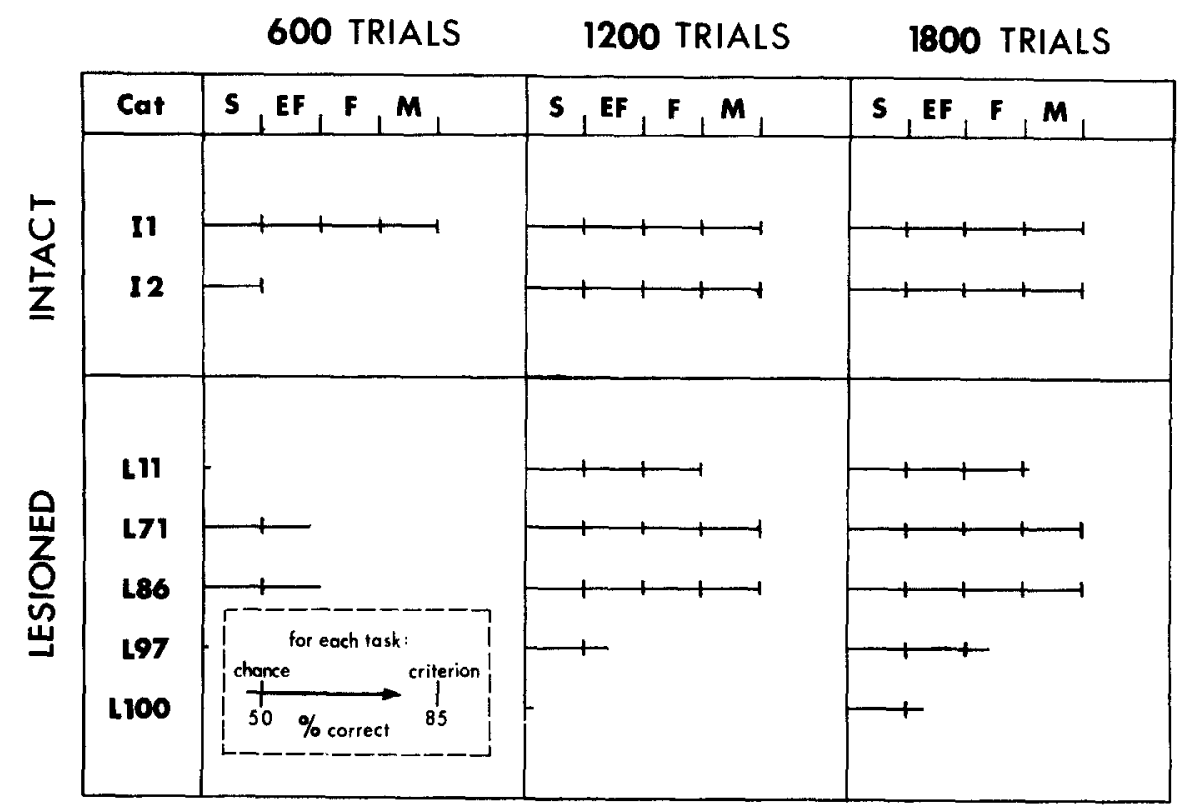

Fig. 3. The progress in performance for two intact cats and 5 cats with dorsal column lesions, at 3 stages in their training. The number of trials does not include trials during which boots were worn. There are 4 tasks: S, extra coarse $v s$. smooth; EF, extra coarse $v s$. extra fine; F, extra coarse vs. fine; $\mathbf{M}$, extra coarse vs. medium. The length of a line in each task is proportional to the cat's accuracy (50-85\% correct), and is determined by his performance in the previous 100 trials (or all trials on that task, if there are fewer than 100). A crossbar on the line indicates the meeting of a criterion (at least $85 \%$ correct for 4 of 5 consecutive days). L97 is included beyond his 600 th trial on extra coarse $v s$. extra fine in spite of failing to meet criterion for that task.

quired for criterion performance on the second task (L97: 1000 trials; L100: 28 trials), (2) 540 trials given to L97 on the third task for which he never reached criterion, and (3) the minimum number of trials which would have been required for L97 and L100 to reach criterion on the third and fourth tasks (i.e., assuming perfect performance). Thus, the statistic is a conservative description of the deficit shown by the cats with the largest lesions.

\section{Roughness discrimination: animals trained preoperatively}

The foregoing experiment tested the effect of DC lesions on learning of a roughness-discrimination task. An additional experiment was designed to determine the effects of DC lesions on retention of that skill. Seven cats were pretrained to discriminate at the second level before they were sham operated (I3, I4, L38) or given DC lesions of $69-100 \%(\mathrm{R} 69, \mathrm{R} 82, \mathrm{R} 86, \mathrm{R} 100)$ and tested 2 weeks later on the first 2 tasks (Fig. 4). The only cat which failed to pass both tests in 1000 trials was the cat with a total DC lesion (Table III). In addition, the pretrained animals with DC lesions show a deficit in the first day's performance ( 20 trials) when compared with shamoperated controls $(t$-test, $P<0.05$ ) (Table IV). 


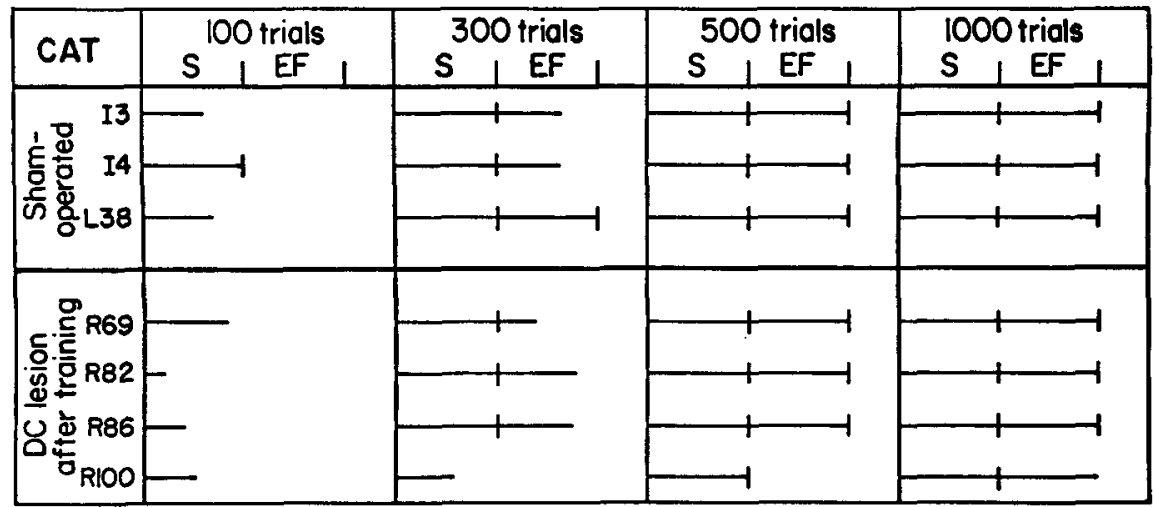

Fig. 4. The progress in performance of 3 sham-operated pretrained cats and 4 pretrained cats given dorsal column lesions. Same conventions as Fig. 3. The two tasks (S, extra coarse vs. smooth; EF, extra coarse $v s$. extra fine) are shown at 4 stages.

In summary, the effect of extensive DC lesions can be demonstrated by careful neurological examination and formal testing of roughness discrimination. However, the positive findings are limited to animals with nearly complete DC destruction and the deficits in discriminative capacity become apparent only after extensive behavioral testing.

\section{DISCUSSION}

The results show that the effects of DC lesions in cats can be demonstrated by

\section{TABLE III}

THE EFFECTS OF DC LESIONS ON THE RETENTION OF ROUGHNESS-DISCRIMINATION SKILL

The number of trials required for each cat to perform at criterion level (at least $85 \%$ correct for 4 of 5 consecutive days) is shown for each discrimination.

Cat Extra coarse vs. smooth Extra coarse vs. extra fine

Cats which had passed criterion for extra coarse $v s$. extra fine, then were sham operated and retested 2 weeks after the surgery

13

I4

L.38

$\begin{array}{ll}160 & 200 \\ 100 & 400 \\ 180 & 100\end{array}$

Cats which had passed criterion for extra coarse $v$ s. extra fine while intact, then were given dorsal column lesions and retested 2 weeks after surgery

$\begin{array}{lll}\text { R69 (I2) } & 140 & 280 \\ \text { R82 (I1) } & 181 & 260 \\ \text { R86 (I6) } & 160 & 280 \\ \text { R100 (I7) } & 500 & *\end{array}$

* The cat was unable to perform at criterion within the 600 trials allowed. 
TABLE IV

INITIAL PERFORMANCE ( $\%$ CORRECT) FOR EACH DISCRIMINATION

Cat

First day on new task (20 trials)

\begin{tabular}{ll}
\hline Extra coarse & Extra coarse \\
vs. smooth & vs. extra fine
\end{tabular}

First 100 trials on new task (5 days)

vs. smooth vs.extrafine vs. smooth vs. extrafine

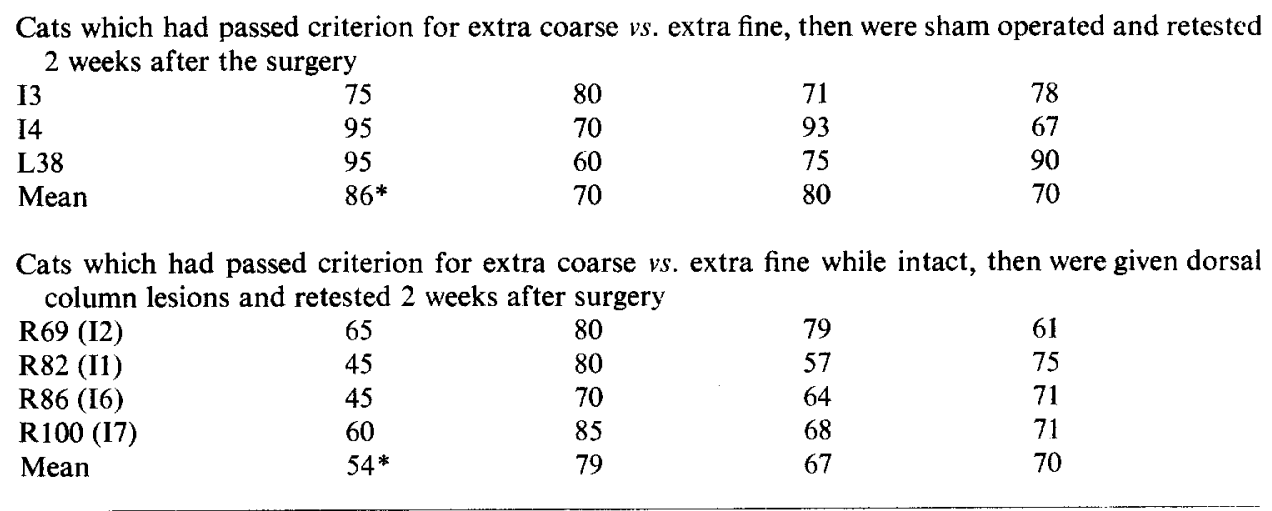

$* t=3.0, P<0.05$.

neurological examination and roughness-discrimination tests, but to show significant effects, $90 \%$ of the cervical dorsal columns must be destroyed. In this study, such lesions were estimated to involve over $90 \%$ of the DC fibers transmitting information from the paws (Table I).

Schiff ${ }^{20}$ reported in 1894 that his dogs with DC lesions frequently turned their feet over with the dorsum downward, as noted for 3 of our cats with lesions greater than $85 \%$. In both studies, this abnormality decreased with time, but it never completely disappeared in the cats. Although there were cats with total DC lesions as large as $86 \%$ that did not have deficits revealed by the neurological examinations, all cats with lesions greater than $90 \%$ did show deficits on these tests. The deficit shown most consistently was an inability to land without stumbling when they were dropped. These results are in agreement with the observations by Melzack and Bridges $^{15}$ on cats with near-total DC lesions.

The cats with large (greater than $90 \%$ ) DC lesions also showed conspicuous deficits in learning or retaining roughness-discrimination skill after DC section. This deficit is probably not related to the cats' inability to associate the stimulus with its meaning in the test situation because the deficit was revealed only in the more difficult tasks. Motivational changes are unlikely contributing factors because the cats performed with the same latency as intact cats. There was no motor impairment which affected performance in the task. The control observations with boots also indicate that the cause of deficits in the roughness-discrimination task is a change in effective somatic input. Finally, it is unlikely that damage to the lateral or dorsolateral columns significantly influenced these results because the cat with a bilateral dorsolateral 
lesion (DL) failed to show neurological deficits and, in cats with complete or nearly complete DC lesions, sparing of lateral column fibers was not associated with improved performance on roughness discrimination even after pretraining ( $R 100$, vs. L100, L97 or R86).

The study of Kitai and Weinberg ${ }^{11}$ is similar to ours except that none of their cats had $90 \%$ DC lesions. In addition, their cats were permanently blinded and thus might have developed increased tactile discriminative capacity through weeks of experience without visual cues. In Kitai and Weinberg's study, the cats were trained for an equal number of trials on a given discrimination, regardless of performance. The training procedure we employed insured that each subject was given the opportunity to learn the first discrimination problem so that performance on subsequent tasks could be compared across animals. Since each subject had learned the basic problem, failure on the more difficult discriminations is more likely to be due to sensory incapacity than an inability to learn for reasons unrelated to somesthetic deficiencies. This is confirmed by the results of lesions after preoperative training, showing that only the cat with a total DC lesion failed to pass both discrimination tasks.

\section{Interpretation of results}

The results are consistent with the hypothesis that there is sufficient redundancy in the information transmitted via the dorsal column that a DC lesion has to be nearly complete before sensory deficits appear. Cats retain a remarkable level of visual capacity, for example, following lesions destroying over $90 \%$ of the optic tract? The primary evoked potential in somatosensory cortex is changed very little by greatly reducing the number of peripheral nerve fibers stimulated ${ }^{1}$, but we do not know how such electrophysiological measures of functional integrity relate to behavioral capacity. The redundancy argument is supported by the observation (Table I) that nearly complete DC lesions could leave intact a fraction of the fibers innervating the fore or hindpaws. On the basis of the data available, however, it is not possible to prove or disprove the redundancy hypothesis.

Wall ${ }^{24}$ has suggested that the dorsal columns are important in the orientation of an animal toward sensory information traveling in other spinal pathways. In discussing the proposition that alternate spinal pathways can functionally substitute for the dorsal columns, Wall ${ }^{24}$ presented experimental evidence against the corollary argument: that the dorsal columns should be able to substitute for the alternate pathway. Thus, rats with thoracic cord lesions sparing only the dorsal columns gave no sign of forelimb or head responses to a variety of hindlimb stimuli. On the other hand, combined lesions of the dorsal columns and other ascending pathways result in greater behavioral impairments than either lesion alone ${ }^{4,12,14,18,22 . ~ T a k e n ~ t o g e t h e r, ~}$ these results suggest a 'permissive' or modulating role of the DC system in somesthesis.

It is possible, then, that a refinement of discrimination or other behavioral tests would reveal deficits with smaller DC lesions. We know from electrophysiological experiments that the dorsal columns are a rapidly conducting sensory system with fine resolution of both the spatial and temporal aspects of stimulation. On this basis, 
further search for DC functions might profitably focus on a combination of spatial and temporal dimensions.

SUMMARY

(1) Seven normal cats and 17 cats with dorsal column (DC) lesions of 2-100\% of the total DC cross-sectional area were given neurological tests. Cats with lesions as large as $86 \%$ showed no deficits on neurological examination. All cats with lesions greater than $90 \%$ showed signs of neurological impairment.

(2) Six cats with high cervical DC lesions were compared with 4 intact controls in a roughness-discrimination task of 4 graded levels. Two cats with $97 \%$ and $100 \%$ destruction of their total DC cross-sectional area failed to reach criterion on the second discrimination level; cats with $38-86 \%$ DC lesions learned the highest discrimination grade as quickly as intact controls.

(3) DC lesions of $69-86 \%$ in pretrained cats failed to produce evidence of lasting postoperative deficits when compared with pretrained, sham-operated controls; however, a cat with a $100 \%$ lesion failed to reach criterion on the second discrimination level.

(4) The results show that the effects of DC lesions in cats can be demonstrated by neurological examination and roughness-discrimination tests. However, to show significant effects, at least $90 \%$ of the dorsal columns must be destroyed; in this study, such lesions were estimated to involve over $90 \%$ of the DC fibers transmitting information from the paws. Although these findings suggest a high level of functional redundancy within the DC system, alternative views of DC function and its analysis are suggested.

\section{ACKNOWLEDGEMENTS}

The authors are indebted to Mr. John Matthews for technical assistance.

This investigation was supported in part by Public Health Grant NS 06588 and Grant TOGM 353-11.

This article summarizes part of a doctoral dissertation submitted by P. J. K. Dobry at the University of Michigan.

\section{REFERENCES}

1 Amassian, V. E., Fiber groups and spinal pathways of cortically represented visceral afferents, J. Neurophysiol., 14 (1951) 445-460.

2 Boshes, B., AND Padberg, F., Studies on the cervical spinal cord of man. Sensory pattern after interruption of the posterior columns, Neurology (Minneap.), 3 (1953) 90-101.

3 CoOx, A. W., AND Browder, E. J., Functions of posterior columns in man, Arch. Neurol. (Chic.), 12 (1965) 72-92.

4 DeVito, J. L., AND Ruch, T. C., Central pathways subserving weight discrimination in monkey, Fed. Proc., 15 (1956) 48-49.

5 DeVito, J. L., Ruch, T. C., and Patton, H. D., Analysis of residual weight discriminatory ability and evoked cortical potentials following section of dorsal columns in monkeys, Indian $J$. Physiol. Pharmacol., 8 (1964) 117-126. 
6 Diamond, I. T., RANDall, W., ANd Springer, L., Tactual localization in cats deprived of cortical areas SI and SII and the dorsal columns, Psychon. Sci., 1 (1964) 261-262.

7 Galambos, R., Norton, T. T., and Frommer, G. P., Optic tract lesions sparing pattern vision in cats, Exp. Neurol., 18 (1967) 8-25.

8 GellermanN, L. W., Chance orders of alternating stimuli in visual discrimination experiments, J. genet. Psychol., 42 (1933) 206-208.

9 Gilman, S., and Denny-Brown, D., Disorders of movement and behavior following dorsal column lesions, Brain, 89 (1966) 397-417.

10 Hekmatpanah, J., Organization of tactile dermatomes $\mathrm{C}_{1}$ through $\mathrm{L}_{4}$, in cat, $J$. Neurophysiol., 24 (1961) 129-140.

11 Kitai, S. T., AND WeInberg, J., Tactile discrimination study of the dorsal column-medial lemniscal system and spino-cervico-thalamic tract in cat, Exp. Brain Res., 6 (1968) 234-246.

12 Levitt, M., and Schwartzman, R., Spinal sensory tracts and two-point tactile sensitivity, Anat. Rec., 154 (1966) 377.

13 LIU, C., Afferent nerves to Clarke's and the lateral cuneate nuclei in the cat, Arch. Neurol. Psychiat. (Chic.), 75 (1956) 67-77.

14 Lundberg, A., AND Norrsell, U., Spinal afferent pathway of the tactile placing reaction, Experientia (Basel), 16 (1960) 123.

15 Melzack, R., ANd Bridges, J. A., Dorsal column contributions to motor behavior, Exp. Neurol., 33 (1971) 53-58.

16 Mettler, F. A., AND Liss, H., Functional recovery in primate after large subtotal spinal cord lesions, J. Neuropath. exp. Neurol., 18 (1959) 509-516.

17 Mountcastle, V. B., Medical Physiology, 12th ed., Mosby, St. Louis, Mo., 1968, p. 1415.

18 Norrsell, U., The spinal afferent pathways of conditioned reflexes to cutaneous stimuli in the dog, Exp. Brain Res., 2 (1966) 269-282.

19 Rabiner, A. M., AND Browder, J., Concerning the conduction of touch and deep sensibilities through the spinal cord, Trans. Amer. neurol. Ass., 73 (1948) 137-142.

20 Schiff, J. M., 1894. Cited by Ferraro, A., AND Barrera, S. E., Effects of experimental lesions of the posterior columns in Macacus rhesus monkeys, Brain, 57 (1934) 307-332.

21 Schwartzman, R. J., AND Bogdonoff, M. D., Proprioception and vibration sensibility discrimination in the absence of the posterior columns, Arch. Neurol. (Chic.), 20 (1969) 349-353.

22 VIERCK, C. J., Spinal pathways mediating limb position sense, Anat. Rec., 154 (1966) 437.

23 WALKer, A. E., AND WeAver, JR., T. A., The topical organization and termination of the fibers of the posterior columns in Macaca mulatta, J. comp. Neurol., 76 (1942) 145-158.

24 WALL, P. D., The sensory and motor role of impulses travelling in the dorsal columns towards cerebral cortex, Brain, 93 (1970) 505-524. 